\title{
Research on Influencing Factors of Teachers' Occupational Well-Being Based on Ecological System Theory
}

\author{
Song Liu \\ School of education, \\ Tianjin University \\ TianJin, China \\ Email: s1950323_@tju.edu.com
}

\begin{abstract}
There are a variety of influencing factors for teachers' occupational well-being, and the research on influencing factors needs to be conducted from the holistic and systematic perspective. Based on Bronfenbrenner's ecological system theory, this paper explores the influencing factors of the teacher's occupational well-being from the four aspects, namely the microsystem, the mesosystem, the exosystem and the macrosystem. In view of the analysis of the influencing factors of the teachers' occupational well-being, this paper puts forward some targeted countermeasures and suggestions, which will provide a new perspective to promote teachers' occupational well-being and to further promote the vitality of the teachers' development.
\end{abstract}

Keywords-Teachers; Occupational well-being; Ecological system theory; Development vitality

\section{INTRODUCTION}

Teachers' occupational well-being means that in their educational work, teachers are able to achieve their full potential freely, satisfy their own material and spiritual needs, realize their professional ideals and their own values freely, and obtain the continuous happiness with their growth of power [1]. With the development of national educational reform, teachers' tasks are becoming more and more burdensome while bringing opportunities for teachers' development. Work pressure and mental stress greatly affect teachers' occupational well-being. However, teachers' occupational well-being can help them to establish a positive attitude in teaching, and make teachers participate in their daily work with full enthusiasm and realize their personal career ideals. It will also help further enhance teachers' teaching quality, students' comprehensive quality and schools' education quality on the basis of teachers' development. And then it improve the overall quality of education in society.

However, few studies use the theoretical model to make tentative analysis of the factors affecting teachers' occupational well-being currently. This paper attempts to explore the influencing factors of teachers' occupational wellbeing from the different levels of the ecological systems based on Bronfenbrenner's ecological systems theory and then puts forward some specific recommendations and suggestions.

\section{THE CONSISTENCY BETWEEN BRONFENBRENNER' $S$ ECOLOGICAL SYSTEMS THEORY AND THE INFLUENCING FACTORS OF TEACHERS' OCCUPATIONAL WELL-BEING}

Ecological systems theory was proposed by the famous American psychologist U Bronfenbrenner. In his theory, the ecological system is divided into the microsystem, the mesosystem, the exosystem and the macrosystem. He used the perspective of ecological systems theory to study the development of children, emphasizing that individuals in development are nested in a series of environmental systems that influence each other. The interaction between ecosystems and individuals affects the development of individuals [2]

Comparing the ecological systems theory with the teachers' occupational well-being, we can see that there is a great similarity and connection between the two. Firstly, from the perspective of research content, the ecological systems theory emphasizes the important influence of the environment on human development. And the related research on the wellbeing also finds that environmental factors have an important impact on the well-being of individuals. Secondly, from the perspective of research value, the ecological systems theory is the theory of human development. Its purpose is to promote the full development of people. The concept of well-being stemming from positive psychology can not only promote the overall development of people, but also promote the realization of social harmony to a certain extent.

\section{EXPLANATION OF FACTORS AFFECTING TEACHERS} OCCUPATIONAL WELL-BEING BASED ON ECOLOGICAL SYSTEMS THEORY

Based on ecological systems theory, we can analyze teachers' occupational well-being from the microsystem, the mesosystem, the exosystem and the macrosystem of teachers' life, and have a systematic understanding of the affecting factors of teachers' occupational well-being. 


\section{A. Factors Affecting Teachers' Occupational Well-being in Microsystem}

The microsystem refers to the relationship and interaction among the developing individuals and the people they are in direct contact with, the environment they are in direct contact with. In the teachers' work environment, the groups directly touched by teachers are students, parents, colleagues, and school leaders. Next this paper will analyze how the environment affects teachers' occupational well-being from four aspects: students, parents, colleagues, and school leaders.

1) The Influence of Students on Teachers' Occupational Well-Being

In the teachers' teaching process, when the students are full of learning motivation or the students' learning goals are consistent with the teachers' teaching goals, teachers can easily get positive feedback from the students. For example, students approve of teachers or students' learning motivation is significantly enhanced and their learning ability is significantly improved under the guidance of teacher. These will increase teachers' sense of professional achievement and thus promote teachers' occupational well-being. On the contrary, it is unfavorable to the teachers' professional wellbeing when the teachers receive negative feedback on the students.

\section{2) The Influence of Parents on Teachers' Occupational} Well-Being

In contact with teachers, most parents are able to coordinate with teachers' work. However, a small number of parents have few contacts with teachers and there are conflicts when they meet, or parents show distrust of teachers, query teachers' teaching ability and are absent from home-school activities. These will reduce teachers' professional efficacy, and thus affect the teachers' occupational well-being.

\section{3) The Influence of Colleagues on Teachers' Occupational} Well-Being

On the one hand, teachers and colleagues work together during work. Teachers obtain social support and energy from their colleagues and can solve problems more effectively and increase their professional efficacy. At the same time, it narrows the psychological distance between teachers and colleagues and enables teachers to gain a sense of belonging to the organization. On the other hand, teachers and colleagues at work will make social comparisons. The asymmetry comparison will cause teachers to have the psychological gap, reduce teachers' satisfaction with their own work and affect their occupational well-being.

4) The Influence of Leaders on Teachers' Occupational Well-Being

Regarding the relationship between teachers and school leaders, Oplatka believes teachers can increase the teachers' enthusiasm for teaching if they are satisfied with the decisions made by school leaders and can be supported by school leaders to some extent [3]. In particular, when leaders provide support for organizational culture and teacher work resources, the enthusiasm and satisfaction of teachers' work will increase, and it will help inspire teachers' positive feelings about work. This is consistent with Aelterman's study that supportive school culture can improve teachers' occupational well-being [4].

\section{B. Factors Affecting Teachers' Occupational Well-being in Mesosystem}

The mesosystem refers to the interaction of various microsystems. The mesosystem in which the teacher is located refers to the interaction between the various microsystems that are in touch with the teacher and it affect the teachers' occupational well-being. The horizontal relationship mainly refers to the relationship between students and students, the relationship between parents and parents, the relationship between colleagues and colleagues, and the relationship between leaders and leaders. The longitudinal relationship mainly includes the relationship between parents and students, colleagues, school leaders, and the relationship between students and colleagues, school leaders. Bronfenbrenner believes that development may be optimized if there is a strong positive link between microscopic systems. On the contrary, it may have negative consequences [2]. According to the direction of the influence of mesosystems on the teachers' occupational well-being, they can be divided into positive mesosystems and negative mesosystems.

The active mesosystem means that the microsystems of students, parents, colleagues, and leaders are in a dynamic and harmonious state. This kind of positive connection will be passed on to teachers so that teachers can receive support and energy in their work, which will facilitate the advancement of the teaching process and increase the job satisfaction of teachers, thus enhancing teachers' occupational well-being.

The negative mesosystem means that the microsystems of students, parents, colleagues, and leaders are in a state of disharmony. This tense connection will also be passed on to teachers. For example, when the relationship between leaders and colleagues is tense and leaders criticize and even abuse other colleagues, they will increase the teachers' emotional pressure and affect their occupational well-being.

\section{Factors Affecting Teachers' Occupational Well-being in Exosystem}

Exosystem refers to an environment system that does not actively participate in the individual development process. However, this level of system can have an indirect effect on individuals through the environment and events. As far as teachers' occupational well-being is concerned, it mainly includes the schools' educational level, the schools' community, and so on.

1) The Influence of Schools' Educational Level on Teachers' Occupational Well-Being.

Teachers work in high-level and demonstration-level schools, which will consciously and unconsciously increase teachers' awareness of their professional values and improve their occupational well-being. 
2) The Influence of Schools' Community on Teachers' Occupational Well-Being.

The educational resources in the community where the school is located are rich, teachers have a high degree of convenience when they work, and their own job involvement is also increased. Their occupational well-being will increase, too. In addition, teachers can communicate and connect with other schools in the community. In this process, teachers can increase their professional skills, improve their job selfefficacy, and stimulate positive emotions in their work.

\section{Factors Affecting Teachers' Occupational Well-being in Macrosystem}

The macrosystem includes various low-level microsystems, the mesosystems, the exosystems as well as the entire social organization, institution, and culture. Specifically, it mainly affects teachers' occupational well-being from aspects such as teachers' professional reputation and national policies.

1) The Influence of Teachers' Professional Reputation on Teachers' Occupational Well-Being.

In the context of Chinese culture, teachers have always been regard as great and holy professions. The higher professional reputation of teachers can stimulate their enthusiasm for work, enhance teachers' sense of professional belonging and professional values, and promote teachers' occupational well-being. However, following the respect of teachers, teachers are pushed onto the altar and become "sages" who cannot make mistakes. Borja found that the expected teachers' skills in social culture have a great influence on teacher happiness [5]. The society puts forward high expectations for teachers, and even slogans such as "only poorly-teaching teachers and no poorly-learning students" have increased the pressure on teachers, which is not conducive to the acquisition and promotion of teachers' occupational well-being.

2) The Influence of National Policies on Teachers' Occupational Well-Being.

For example, Article 20 of the "Law of the People's Republic of China on Teachers" stipulates: The average wage level of teachers should not be lower or should be higher than the average salary level of the state civil servants, and gradually increase. And establish a normal promotion and salary increasing system [6]. As teachers are living people, their happiness is closely related to their career income and job promotion. National policy protects teachers' income and promotion from the legal level, thereby stimulating teachers' professional feelings and professional values. And further improve the level of occupational well-being of teachers.

\section{SUgGeStions ON PROMOTING TEACHERS' OCCUPATIONAL WELL-BEING BASED ON ECOLOGICAL SYSTEMS THEORY}

Teachers' occupational well-being is derived from the organic interaction between teachers themselves and different levels of environmental systems. This requires us to have a holistic view and a systematic perspective when we look at the issues related to teachers' occupational well-being.

The current research pays more attention to the effect of single environmental factor on the teachers' occupational well-being, and ignores the interaction between different levels of environmental systems and teachers. Over time, the essence of the problem has not yet been solved. Each system has its own role in improving the teachers' occupational wellbeing. The lagging effect or the mismatched effect between systems and systems may weaken the effect of each system on promoting teachers' occupational well-being. Therefore, improving teachers' occupational well-being needs to balance the effects of various systems. So that the systems can move in a coordinated direction and promote the sustained development of teachers' occupational well-being. Based on the above analysis, this paper proposes the following suggestions for improving teachers' occupational well-being:

\section{A. Skills Driven - Working Competently}

Well-being comes from personal efforts. Teachers must have a certain amount of professional knowledge and professional skills in order to take targeted strategies to solve many problems when they communicate with students and parents. This requires teachers to focus on strengthening their own professional skills training, planing scientific and rational professional development, getting out of the their own development difficulties, constantly enriching themselves and mastering advanced teaching ideas and teaching methods. Teachers are also required to stimulate students' enthusiasm for learning and learning motivation, to communicate effectively with parents in a timely manner. As a result, teachers cultivate more outstanding students, get positive feedback from parents and students and promote personal well-being.

\section{B. Humanity Management - Working powerfully}

In order to improve teachers' occupational well-being, school leaders should establish "people-oriented" management concept and give full play the leading role of teachers. First of all, school leaders should analyze the individual differences among teachers to maximally stimulate teachers' the potential of development, allow teachers to work in the positions where they are good at so that teachers can win the support and approval of colleagues and leaders. In this way, the enthusiasm of teachers' development can be fully mobilized, and their well-being in work will increase accordingly; then, in the teachers' job evaluation system, fair and differentiated evaluation should be carried out. This can reduce teachers' psychological gaps. Teachers are more likely to generate attribution and attachment to the group and promote cooperation and communication between teachers and colleagues; finally, the channels for communication between teachers and school leaders should be broadened. Making teachers work harder in a good work environment can enhance 
their professional efficacy and inspire positive emotions in their work.

\section{Community Support - Working interactively}

First of all, teachers can make full use of the resources of the community where the school is located to set up and arrange their own courses and communicate effectively with their communities. Then, teachers can strengthen communication with other schools in the community and obtain professional support and psychological support. . In the above interaction, teachers can achieve their own occupational well-being.

\section{Building Atmosphere - Working Securely}

There is no doubt that the macrosystem has a decisive influence on the teachers' occupational well-being. First of all, while society is building the public opinion atmosphere of respecting teachers and valuing education, it can't "deify" teachers. The masses cannot always hold a magnifying glass to treat the work of teachers. Parents should respect teachers on the basis of fully understanding the work of teachers. The media should also play a role of public opinion guidance and give teachers necessary understanding and tolerance. In addition, the state must strengthen its support for teachers' income and promotion. The state should also improve relevant laws and regulations, and legal services should be targeted at a broader group of basic education teachers. Under the overall situation of ruling the country by the law, it is necessary to rule education by the law and provide legal protection for teachers.

\section{CONCLUSION}

All in all, under the current national background of advocating people-oriented and building the harmonious society, the pursuit of happiness has become the common vision of the whole society. The ultimate ideal of education is also to cultivate a happy person for the society. Only teachers with a high level of occupational well-being can educate happy students. The improvement of teachers' occupational well-being has important significance for students, teachers, schools and even the entire society. This paper, based on the ecological systems theory, looks back on teachers' occupational well-being through environmental factors, emphasizing the holistic view and the systematic perspective of the environment, and provides useful theoretical guidance for exploring the improvement of teachers' occupational wellbeing.

\section{REFERENCES}

[1] Cai Lingli. Influencing Factors and Enhancement Strategies of College Teachers' Occupational Well-being [J]. Educational Theory and Practice, 2010(36):39-41. (In Chinese)

[2] Bronfenbrenner U. The ecology of human development: [M]. Harvard University Press, 1979.

[3] Oplatka I. Managing Emotions in Teaching: Toward an Understanding of Emotion Displays and Caring as Nonprescribed Role Elements [J]. Teachers College Record, 2005, 109(6):1374-1400.

[4] Aelterman A, et al. The well-being of teachers in Flanders: the importance of a supportive school culture [J]. Educational Studies, 2007, 33 (3): 285-297.

[5] P. Borja, Amanda. Phenomenology of education stakeholders about teacher competencies in New Orleans and SriLanka: Implications for teacher well-being [D]. Tulane University, 2012.

[6] Law of the People's Republic of China on Teachers [EB/OL]. (2005-0525). HTTP: // WWW. gov. c/ banshi/ 2005-05/25/ content_937. htm. (In Chinese) 